\title{
Dificultades de atención y niveles de aprendizaje en estudiantes de Educación General Básica (EGB)
}

\section{Attention difficulties and levels of learning in Basic General Education students (GEB)}

\author{
Ángel Arrobal, Lenin Mendietą2 Angélica Gaibor, Diego Chacón, Yolanda Cuenca
}

\section{RESUMEN}

El objetivo de estudio fue diagnosticar y relacionar las dificultades de atención con los niveles de aprendizaje, la metodología utilizada fue: investigación no experimental; cuanti-cualitativa; de tipo histórica-lógica, descriptiva, exploratoria comparativa; se arrimó al paradigma socio crítico; fue seccional; la población fueron, los alumnos de la Unidad Educativa "Pío XII", la muestra de 44 estudiantes del Octavo año de EGB, seleccionada al azar, sin discriminación de género; la variable dependiente fueron los niveles de aprendizaje, y la independiente las dificultades de atención; las técnicas e instrumentos utilizados fueron: Observación directa, entrevistas y test. Los resultados arrojados confirman la existencia de casos de déficit de atención y una relación entre esta y los niveles de aprendizaje en los casos estudiados. Las conclusiones son afines a la literatura especializada

Palabra clave: Estudiantes, dificultades de atención, niveles de aprendizaje, educación general básica

\section{ABSTRACT}

The objective of this study was to diagnose and fix attention difficulties with learning levels, the methodology used was: no experimental research; quantitative and qualitative; historical-type logic, descriptive, comparative exploration; He pulled to critical socio paradigm; It was sectional; the people were, the students of the Educational Unit "Pius XII", the sample of 44 students of the eighth year of EGB, selected at random, without gender discrimination; the dependent variable was learning levels, and independent attention difficulties; the techniques and instruments used were: direct observation, interview and test. Thrown results confirm the existence of cases of attention deficit and a relationship between this and learning levels in the cases studied. The findings are similar to the literature

Keywords: Students, attention difficulties , learning levels, basic general education

\footnotetext{
1. Docente Investigador de la Pontificia Universidad Católica del Ecuador, sede Santo Domingo, aearrobac@pucesd.edu.ec 2. Docente Investigador de la Universidad Estatal de Guayaquil, facultad de medicina, leninbyronmt@gmail.com

3. Estudiantes Investigadores de la Carrera de Ciencias de la Educación, apgaiborg@pucesd.edu.ec, dchacona@pucesd.edu.ec ykcuencaa@pucesd.edu.ec
} 


\section{INTRODUCCIÓN}

Para poder establecer relación entre las dificultades de atención con los niveles de aprendizaje, a más de establecer un diagnóstico que determine la hoja de ruta a seguir para el tratamiento del problema, es necesario el empoderamiento del objeto desde la literatura especializada, así según un estudio (Martí, 2009), las dificultades de atención se convierten en un proceso psicológico (la percepción, la memoria o el aprendizaje) que actuando en conjunto, se traduce en la adaptación continua al medio ambiente de parte de los seres humanos.

Los fallos en el proceso global de atención generan a su vez una disminución en la mencionada adaptación, así se genera dudas si el sistema educativo se ha equivocado a la hora de establecer los procesos de inter aprendizaje en las aulas escolares o existen factores exógenos al entorno escolar que determinan la atención de los alumnos en la educación general básica a nivel mundial, regional o local.

Es muy importante desarrollar la atención en los niños principalmente, convirtiéndose el medio ambiente en el eje condensador de la atención y su desarrollo, sin embargo es tan inmenso el entorno en el que habitamos, que a veces resulta difícil lograr que la atención sea el vector por donde transiten los educandos.

Existen diferentes tipos de atención, así (Grimley, 1992) indica que la atención suele ser selectiva, sostenida y dividida; la primera son habilidades de una persona para responder a los aspectos esenciales de una tarea o situación; la sostenida son habilidades que permiten a un individuo mantenerse consciente de los requerimientos de una tarea y ocuparse de ella por cierto tiempo y; la tercera permiten que en tareas de aritmética o lecto-escritura se manifiesten los dos tipos de atención.

Existen síntomas inequívocos que permiten diagnosticar la falta de atención en los niños, así en el estudio (Sánchez, 1998) sobre la aparición de síntomas, se puede distinguir tres: distracción o falta de atención propiamente dicha; impulsividad; hiperactividad. Las mismas que a su vez presentan características particulares, haciendo más difícil su diagnóstico.

Profundizando el estudio, se puede visualizar manifestaciones de tipo conductual-académico en los niños y jóvenes que tienen diferentes patologías de deficiencia de atención, así (Lahey, 1984) manifiesta que desde el punto de vista comportamental y académico, los sujetos con TDAH - trastornos de déficit de atención con hiperactividad- padecen más problemas de conducta, agresividad, e irresponsabilidad en tareas; por el contrario, quienes padecen trastornos de déficit de atención sin hiperactividad son percibidos como más ansiosos, perezosos e introvertidos. De igual forma los alumnos que presentan un grado de deterioro académico mayor, son los de déficit de atención con hiperactividad.

$\mathrm{Al}$ abordar la relación entre dificultades de la atención y niveles de aprendizaje, es necesario aclarar el concepto de aprendizaje, (Chavarría, 2007) manifiesta que es el proceso de elaboración y construcción del contenido cognitivo, pasa por descubrir, seleccionar, organizar y la correlación de la información que recibe con sus esquemas mentales y conocimientos previos, la transforma en una adquisición cognitiva personal; se trata de un aprendizaje significativo.

Los aprendizajes se pueden evaluar de distintas maneras, obedeciendo a la competencia que se desea evaluar, en la EGB se está generalizando el uso de pruebas de conocimientos específicos. El rendimiento académico, por otro lado, es el resultado cualitativo o cuantitativo alcanzado por los alumnos en una institución educativa, en función del logro de los objetivos y aprendizajes, para un período escolar.

$\mathrm{Al}$ analizar la situación problemática de las dificultades de atención desde una concepción holística, se presenta de manera tangible carac- 
terísticas como: inmediatez, atención dividida, superficialidad y vértigo; siendo lógicamente, nuestros niños y adolescentes quienes absorben más fácilmente dichas características.

En el contexto educativo ecuatoriano, en la EGB es preciso entonces diagnosticar las dificultades de atención y la relación de esta con los niveles de aprendizaje en lenguaje y matemáticas; tipos, porcentajes e incidencia por género.

\section{MATERIALES Y MÉTODOS}

El diseño de la investigación es no experimental; cuanti-cualitativa, así se recogieron, tabularon, graficaron, interpretaron y contextualizaron los datos obtenidos en el escenario natural.

Además, la investigación es de tipo histórica-lógica, descriptiva, exploratoria comparativa y correlacional. Se arrima a la vera del paradigma socio crítico, ya que busca solventar un problema social y cambiar las formas de abordar el objeto para la trasformación de la persona humana, desde el criticismo científico del objeto es transversal o seccional.

\section{Población/ muestra:}

La población de estudio fueron los alumnos de la Unidad Educativa "Pío XII", la muestra fueron 44 estudiantes del Octavo año de EGB, la muestra fue seleccionada al azar, sin discriminación de género, la edad fluctuó entre 11 y 12 años.

\section{Variables:}

La variable dependiente fueron los niveles de aprendizaje, y la independiente las dificultades de atención; mismas que se tuvieron en cuenta en el análisis correlacional.

\section{Técnicas e instrumentos:}

Las técnicas y sus instrumentos utilizados fueron: Observación directa, entrevistas y test.

Para diagnosticar la variable "dificultades de atención" se aplicó a los profesores del grupo de estudiantes seleccionado, el instrumento
"Escala para la evaluación del trastorno por déficit de atención con hiperactividad (EDAH)", usado en varios estudios (Blásquez-Almería, G, Joseph-Muné, D., y otros. 2005) cuestionario de Gilbert (1982) y las escalas para Padres y Maestros de Conners.

La escala EDAH permite evaluar los rasgos principales del TDAH de una manera sencilla y objetiva a través de la información aportada por el profesor, quien a diferencia de los padres, puede comparar al alumno con el resto del grupo y ser más objetivo; el ámbito de aplicación es para sujetos de los 6 a 12 años.

Las respuestas a cada ítem se valoran en una escala de 0 a 3 puntos de acuerdo con el grado de frecuencia con que se percibe la conducta descrita. Cada puntuación se transforma en su puntuación percentil correspondiente; mediante puntos de corte se establecen los niveles de riesgo de padecer el trastorno.

Se proponen 20 ítems; las 5 sub escalas a evaluarse son: Déficit de atención, hiperactividad, trastornos de conducta, hiperactividad más déficit de atención y el de tipo combinado.

Una vez hecho el pre-diagnóstico de los estudiantes, se les aplicó entrevistas personalizadas por parte de un profesional en Psicología Clínica y Educativa, con el fin de complementar los resultados del test y detectar los alumnos con esta patología.

Para evaluar la variable "niveles de aprendizaje", se analizaron dos de sus estadísticos del proceso de aprendizaje escolar (mediciones numéricas que describen características de una muestra), como son: el rendimiento académico y el dominio de conocimientos específicos.

Para analizar el rendimiento académico se usó el cuadro de calificaciones proporcionado por el departamento de Secretaría de la institución, correspondiente a las asignaturas de matemática y lenguaje, durante el primer quimestre y segun 
do parcial del segundo quimestre (hasta la fecha en la que se realizó la investigación) del año lectivo 2014-2015.

Para evaluar los conocimientos específicos se aplicaron pruebas de lenguaje y matemática, basadas en destrezas con criterios de desempeño e indicadores esenciales de evaluación y que fue elaborada a partir del documento "Guía para Docentes de Educación General Básica”, del Ministerio de Educación, para el grupo escolar sujeto de estudio.

Se procedió a establecer la correlación entre variables, en dos momentos: primero la correlación entre dificultades de atención y rendimiento académico y entre dificultades de atención y dominio de conocimientos específicos.

\section{Técnicas de análisis de datos:}

Para la ordenación, interpretación y análisis de los resultados obtenidos de las distintas variables, cálculo del tipo de correlación entre variables y de las ecuaciones de regresión, se usaron hojas de cálculo y siguieron los métodos de la estadística descriptiva.

Prueba experimental de erosión con el ácido cítrico, durante 24 horas evaluando cada tres horas la disolución del troquel de ionómero de vidrio inmerso en la solución.

\section{RESULTADOS}

\section{Tabla $\mathrm{N}^{\circ} 1$. Media aritmética y porcientos de las puntuaciones del EDAH, según tipo de trastorno y género} (la muestra es de 44 estudiantes, de los cuales 23 son varones y 21 son mujeres)

\begin{tabular}{|c|c|c|c|c|c|c|c|c|c|c|c|c|c|c|}
\hline \multirow{2}{*}{ 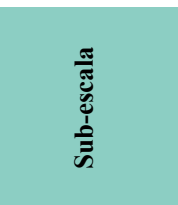 } & \multirow{2}{*}{ 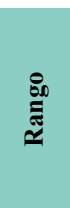 } & \multirow{2}{*}{ 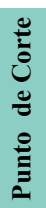 } & \multicolumn{3}{|c|}{$\begin{array}{l}\text { Medias de las } \\
\text { puntuaciones }\end{array}$} & \multicolumn{3}{|c|}{$\begin{array}{c}\text { Porcentaje de incidencia } \\
\text { de las puntuaciones } \\
\text { (posibles trastornos) }\end{array}$} & \multicolumn{3}{|c|}{$\begin{array}{l}\text { Cantidad de estudiantes } \\
\text { que tendrían el trastorno }\end{array}$} & \multicolumn{3}{|c|}{$\begin{array}{l}\text { Porcentaje de estudiantes } \\
\text { que tendrían el trastorno }\end{array}$} \\
\hline & & & 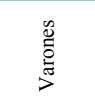 & 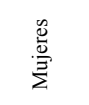 & 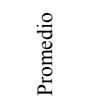 & 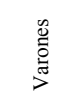 & 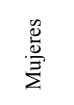 & 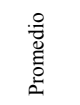 & 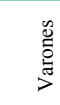 & 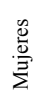 & 퓽 & 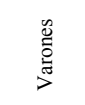 & 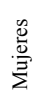 & 范 \\
\hline |Hiperactividad $(\mathrm{H})$ & 0 a 15 & 10 & $4,07 / 15$ & $1,88 / 15$ & $3,07 / 15$ & $27,1 \%$ & $12,5 \%$ & $20,5 \%$ & 2 & 0 & 2 & $4,5 \%$ & $0 \%$ & $4,5 \%$ \\
\hline $\begin{array}{c}\text { Trastorno de } \\
\text { conducta (TC) }\end{array}$ & 0 a 30 & 11 & $5,39 / 30$ & $3,29 / 30$ & $4,39 / 30$ & $17,9 \%$ & $11 \%$ & $14,6 \%$ & 2 & 0 & 2 & $4,5 \%$ & $0 \%$ & $4,5 \%$ \\
\hline $\begin{array}{l}\text { Déficit de atención } \\
\quad \text { (DA) }\end{array}$ & 0 a 15 & 10 & $5,23 / 15$ & $2,15 / 15$ & $3,76 / 15$ & $34,9 \%$ & $14,3 \%$ & $25,1 \%$ & 6 & 0 & 6 & $13,7 \%$ & $0 \%$ & $13,7 \%$ \\
\hline $\begin{array}{l}\text { Hiperactividad } \\
\text { más déficit de } \\
\text { atención }(\mathrm{H}+ \\
\text { DA) }\end{array}$ & 0 a 30 & 18 & $9,29 / 30$ & $4,14 / 30$ & $6,84 / 30$ & $31,0 \%$ & $13,8 \%$ & $22,8 \%$ & 4 & 0 & 4 & $9,1 \%$ & $0 \%$ & $9,1 \%$ \\
\hline $\begin{array}{l}\text { Hiperactividad } \\
\text { más déficit de } \\
\text { atención más } \\
\text { trastorno de } \\
\text { conducta } \\
(\mathrm{H}+\mathrm{DA}+\mathrm{TC})\end{array}$ & 0 a 60 & 30 & $14,6 / 60$ & $7,43 / 60$ & $11,2 / 60$ & $24,3 \%$ & $12,4 \%$ & $18,7 \%$ & 2 & 0 & 2 & $4,5 \%$ & $0 \%$ & $4,5 \%$ \\
\hline
\end{tabular}

Fuente: Test EDAH aplicado a docentes de Octavo "B” de la UEM "Pío XII" 


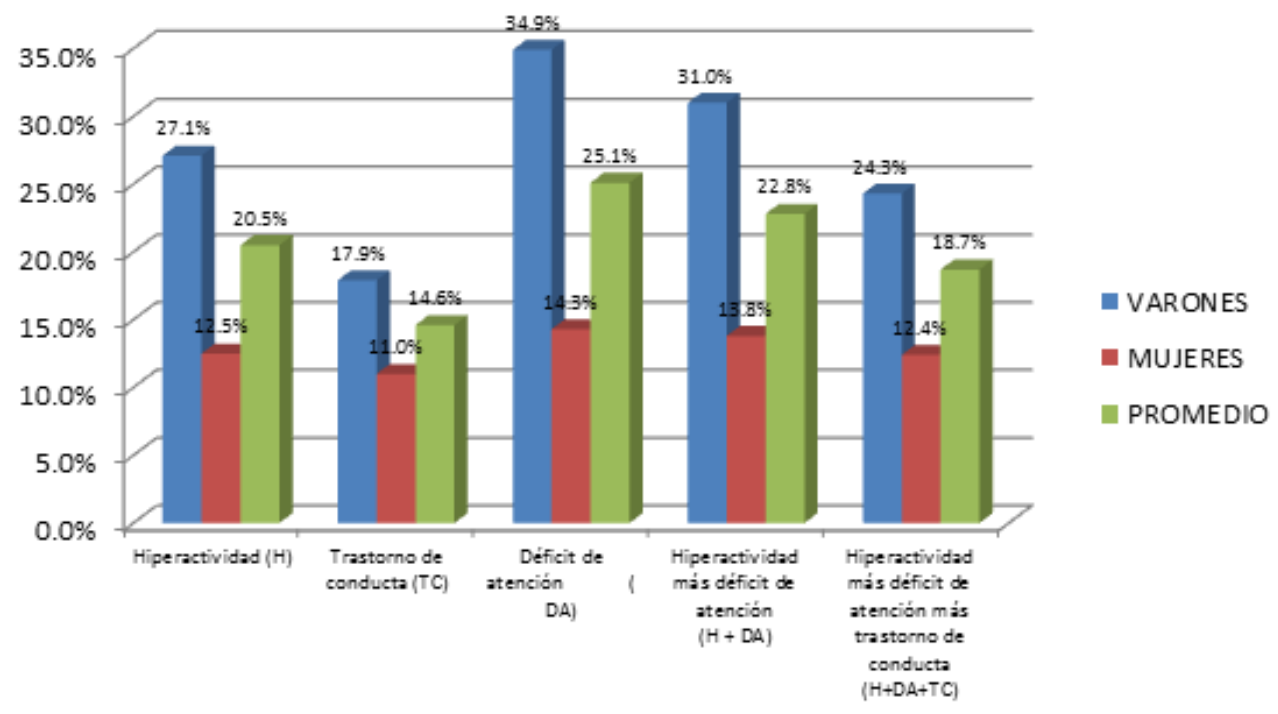

Fuente: Test EDAH aplicado a docentes de Octavo “B” de la UEM “Pío XII”

Gráfico $\mathrm{N}^{\circ} 1$. Porcentaje de incidencia de los posibles trastornos en los estudiantes de Octavo "B" de la UEM "Pío XII".

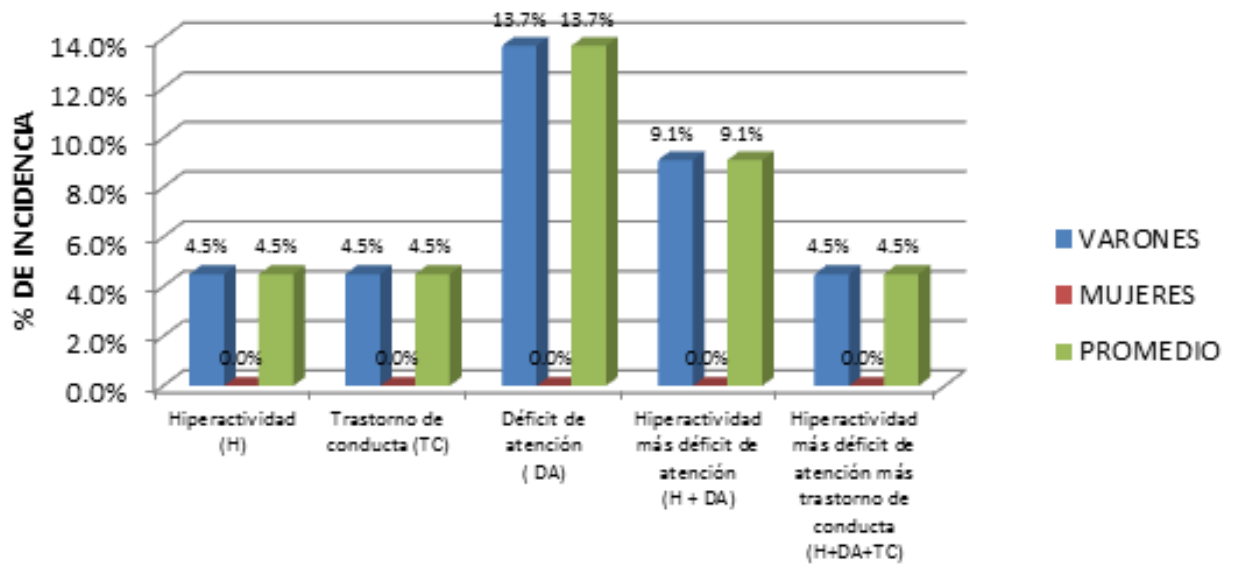

Fuente: Test EDAH aplicado a docentes de Octavo “B” de la UEM “Pío XII”

\section{Gráfico $N^{\circ}$ 2. Porcentaje de estudiantes de Octavo "B" de la UEM "Pío XII" que tendrían el trastorno.}

De la tabla 1 y gráficos 1 y 2 respectivamente, en referencia a los puntajes generales, los trastornos asociados a la atención más frecuentes, según respuesta de los profesores fueron: Déficit de atención $(3,76 / 15$ o $25,1 \%$ con desviación estándar (D.E) de 3,48), hiperactividad más déficit de atención $(6,84 / 30$ o $22,8 \%$ D.E 5,6), hiperactividad más déficit de atención más trastorno de conducta $(11,22 / 60$ o $18,7 \%$ D.E 9,11), hiperactividad sola $(3,07 / 15$ o $20,5 \%$ D.E 2,62$)$ y trastorno de conducta $(4,39 / 30$ o $14,6 \%$ D.E 3,92). 
Tabla $N^{\circ}$ 2. Detalle de las puntuaciones de los estudiantes pre-diagnosticados con déficit de atención (rango de las puntuaciones: 0 a 15; punto de corte: 10)

\begin{tabular}{|cc|}
\hline Estudiante & Puntuación en el test de déficit de atención \\
\hline Estudiante No. 1 & 11,00 \\
Estudiante No. 2 & 12,50 \\
Estudiante No. 3 & 10,50 \\
Estudiante No. 4 & 11,75 \\
Estudiante No. 5 & 10,75 \\
Estudiante No. 6 & 12,50 \\
\hline
\end{tabular}

Fuente: Test EDAH aplicado a docentes de Octavo "B" de la UEM “Pío XII"

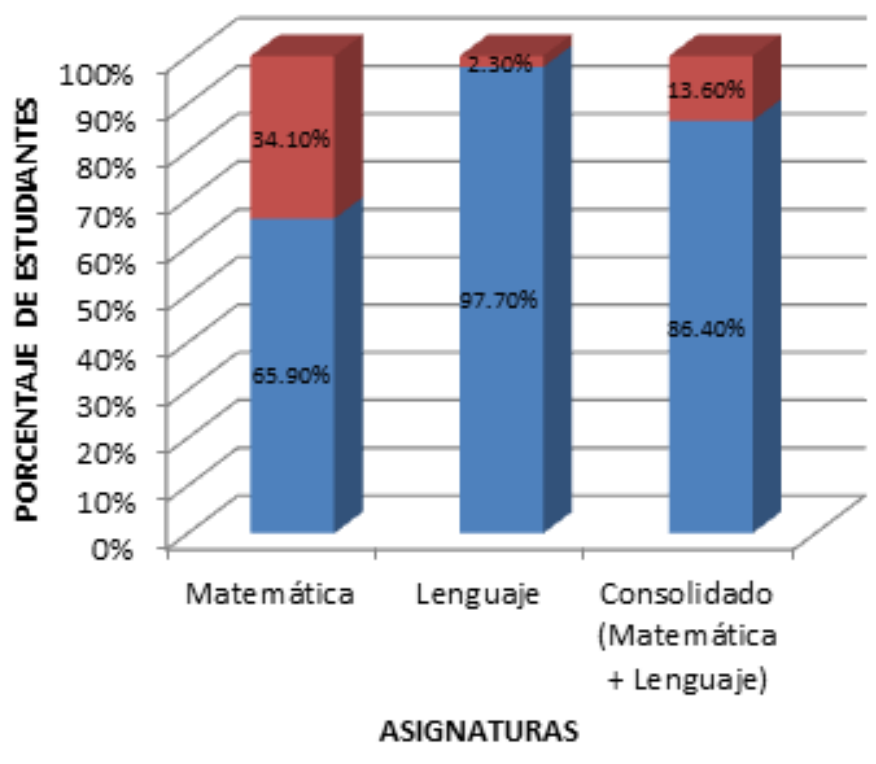

$\%$ DE ESTUDIANTES CON BAJO RENDIMIENTO ESCOLAR (<7)

\% DE ESTUDIANTES CON BUEN RENDIMIENTO ESCOLAR (27)

\section{Gráfico $\mathrm{N}^{\circ}$ 3. Comparación de los porcentajes de estudiantes con bajo y buen rendimiento escolar, por asignaturas $(n=44$; punto de corte: $7 / 10$ puntos)}

El estadístico "rendimiento académico", se obtuvo con los datos proporcionados por Secretaría General de la institución, señalan que la media aritmética en la asignatura de matemática es de $7,56 / 10(75,6 \%)$ con baja dispersión de datos (D.E 1,18).

Los estudiantes que están por debajo del punto de corte (7 puntos) son $15(34,1 \%)$, y que presentan bajo rendimiento.

En la asignatura de lenguaje, la media aritmética del rendimiento académico es de 8,48/10
$(84,8 \%)$ con baja dispersión de datos (D.E 0,95). El número de estudiantes que están por debajo del punto de corte es de apenas uno (2,3\%).

La media aritmética consolidada (matemática más lenguaje) del rendimiento es de 8,02/10 $(80,2 \%)$ D.E 0,95 . El número de estudiantes que están por debajo del punto de corte y que representan bajo rendimiento académico es 6 $(13,6 \%)$. 


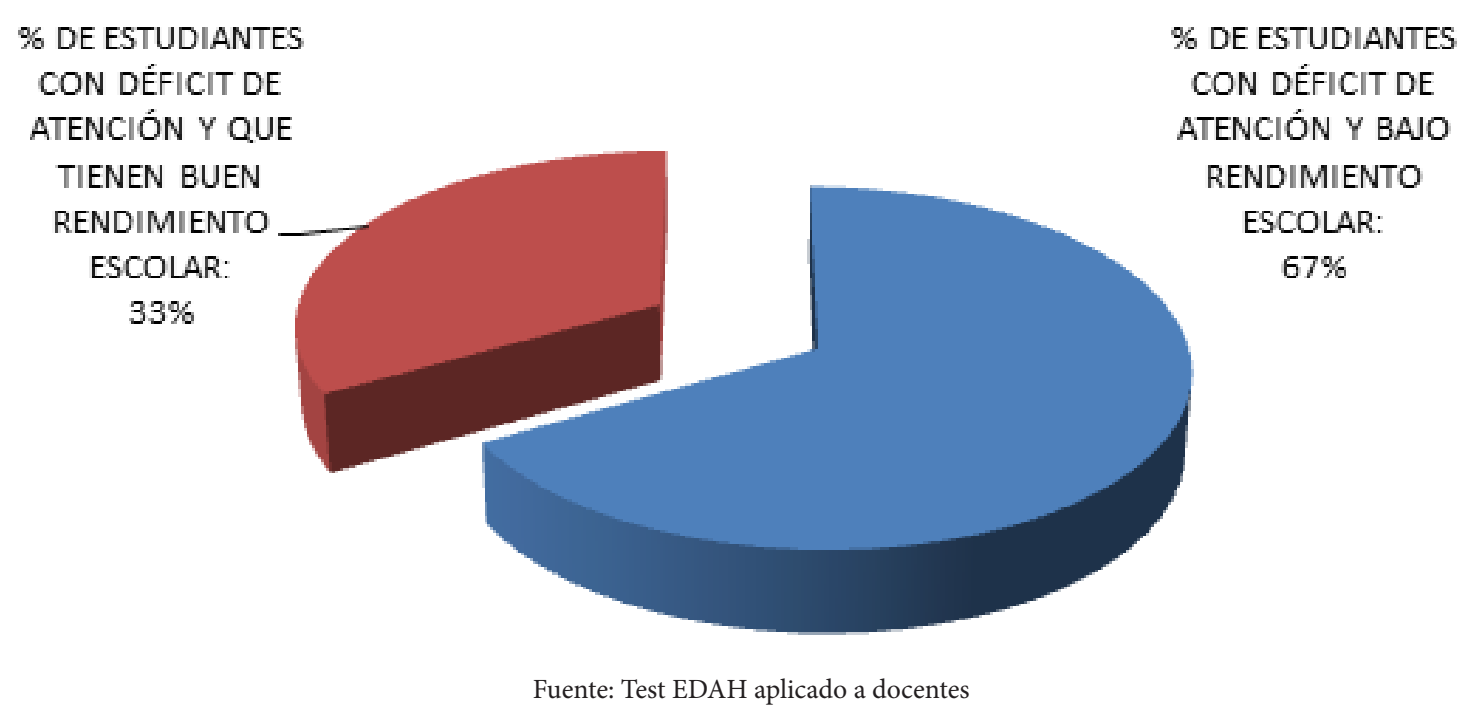

Gráfico $\mathrm{N}^{\circ}$ 4. Porcentaje de estudiantes que presentan déficit de atención y bajo rendimiento escolar $(\mathrm{n}=44)$

Cuatro de seis estudiantes (66,7\%) que presentan pre-diagnóstico de déficit de atención son parte del grupo que tienen "bajo rendimiento escolar" en el consolidado de las dos asignaturas

\section{COMPARACION DE LOS PORCENTAJES DE ESTUDIANTES CON BAJO Y BUEN DOMINIO DE CONOCIMIENTOS ESPECÍFICOS}

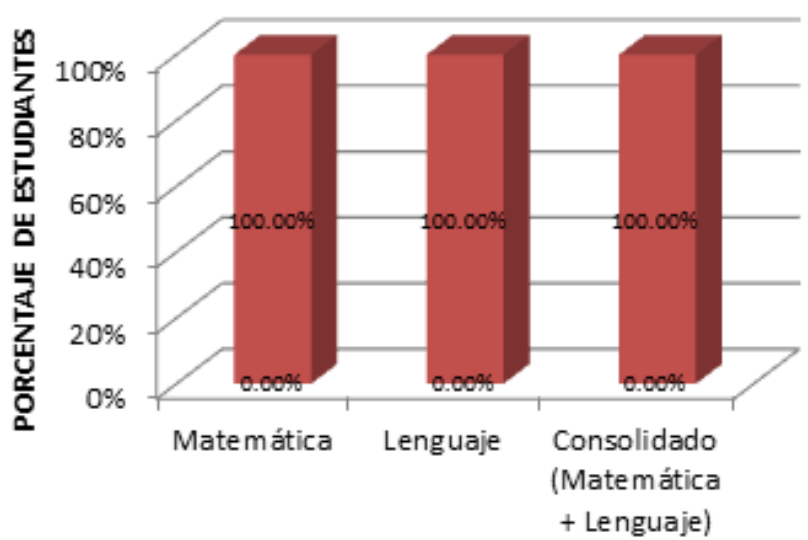

ASIGNATURAS
\% DE ESTUDIANTES QUE NO DOMINA CONOCIMIENTOS ESPECÍFICOS (<7 PUNTOS/10)

— DE ESTUDIANTES QUE DOMINA CONOCIMIENTOS ESPECÍFICOS ( $\geq 7$ PUNTOS /10)

Fuente: Pruebas de conocimientos específicos abalizados por el Ministerio de Educación del Ecuador

Gráfico $N^{\circ}$ 5. Comparación de los porcentajes de estudiantes con bajo y buen dominio de conocimientos específicos, por asignaturas (punto de corte: $7 / 10$ puntos) 
El estadístico "dominio de conocimientos específicos", arrojó resultados son muy bajos: la media aritmética de la prueba de conocimientos específicos de matemática es 1,92/10 (19,2\%) con poca dispersión de datos (D.E 1,31).

El número de estudiantes que están por debajo del punto de corte (7/10) es 44, es decir el 100\% del alumnado del grupo investigado no domina conocimientos específicos de matemáticas.

En la asignatura de lenguaje, la media aritmética de la prueba de dominio de conocimientos específicos es 2,98/10 (29,8\%) con una desviación estándar baja de 1,32.
El número de estudiantes que están por debajo del punto de corte es 44, es decir, también el $100 \%$ del alumnado del grupo investigado no domina conocimientos de lenguaje.

La media aritmética consolidada de las dos pruebas de conocimientos específicos es $2,45 / 10$ $(24,5 \%)$, concluyéndose que el dominio de conocimientos específicos es pésimo de apenas el $24,5 \%$ y una dispersión de datos baja (desviación estándar 1,01).

Todos los estudiantes de la muestra (100\%) están por debajo del punto de corte y presentan bajo dominio de conocimientos en éstas asignaturas.

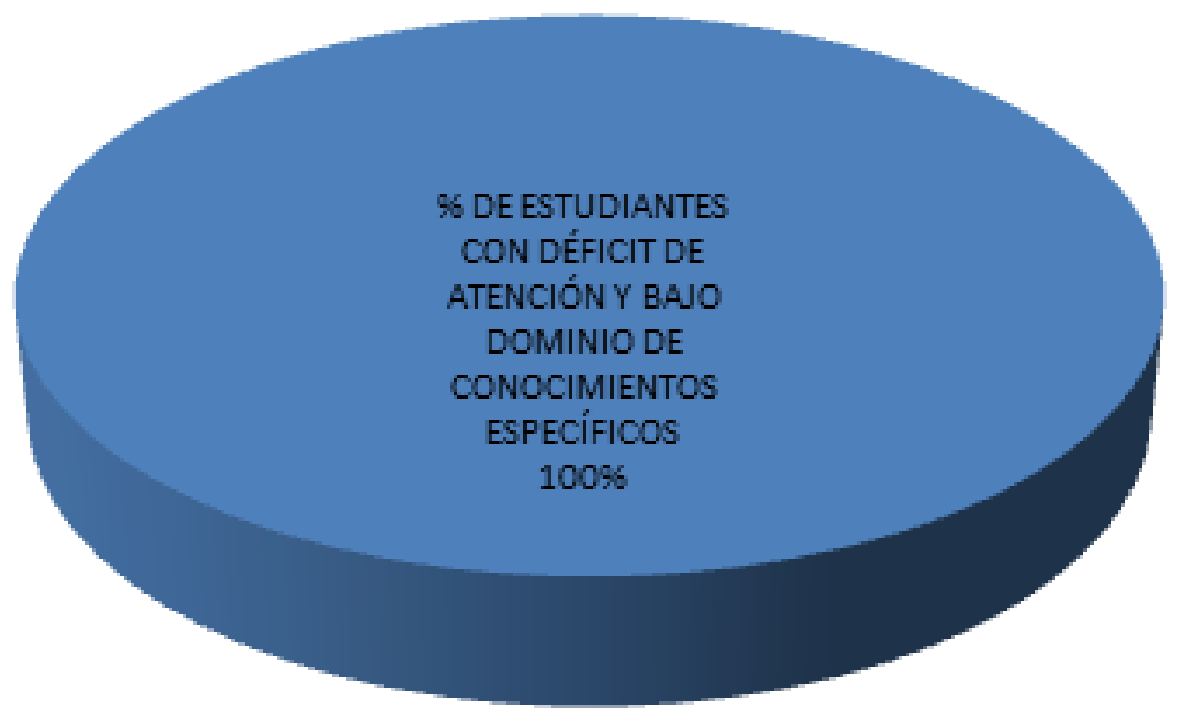

Fuente: Test EDAH y pruebas de conocimientos específicos aplicadas a estudiantes.

\section{Gráfico $\mathrm{N}^{\circ}$ 6. Porcentaje de estudiantes que presentan déficit de atención y bajo dominio de conocimientos específicos en matemática y lenguaje $(n=44)$.}

Además, como existen 6 estudiantes pre-diagnosticados con déficit de atención y todos los estudiantes de la muestra tienen bajo dominio de conocimientos específicos de matemática y lenguaje, por tanto la totalidad de estudiantes que presentan déficit de atención (100\%) presentan bajo dominio de conocimientos específicos; evidenciándose la estrecha relación entre estas dos variables y coincidiendo con lo que señalan los estudios de los distintos autores en este tema. Se muestra en el gráfico: 
Tabla $N^{\circ}$ 3. Detalle de las puntuaciones de los 6 estudiantes pre-diagnosticados con déficit de atención (rango: 0 a 15; punto de corte: 10) y sus puntuaciones del rendimiento escolar (rango: 0 a 10; punto de corte: 7) y de dominio de conocimientos específicos (rango: 0 a 10; punto de corte: 7)

\begin{tabular}{|c|c|c|c|}
\hline Estudiante & Puntuación déficit de atención & Puntuaciones rendimiento escolar & Puntuaciones conocimientos específicos \\
\hline Estudiante No. 1 & 11,00 & 6,33 & 1,72 \\
\hline Estudiante No. 2 & 12,50 & 6,80 & 2,86 \\
\hline Estudiante No. 3 & 10,50 & 7,54 & 1,03 \\
\hline Estudiante No. 4 & 11,75 & 5,39 & 0,79 \\
\hline Estudiante No. 5 & 10,75 & 6,86 & 2,91 \\
\hline Estudiante No. 6 & 12,50 & 7,14 & 2,48 \\
\hline
\end{tabular}

Fuente: Test EDAH aplicado a docentes, Cuadros de calificaciones de estudiantes facilitados por Secretaría y Pruebas de conocimientos específicos

\section{DISCUSIÓN}

Se coincide con el criterio especializado (Martí, 2009), en cuanto a que las dificultades de atención se convierten en un proceso psicológico (la percepción, la memoria o el aprendizaje) que actuando en conjunto, se traduce en la adaptación continua al medio ambiente de parte de los seres humanos.

Los resultados de esta investigación coinciden con el de Hidalgo y Sánchez (2014) cuando señala que el trastorno de déficit de atención con o sin hiperactividad es el trastorno neuro-psico-biológico más frecuente en la edad infanto-juvenil, el grupo etario de nuestro estudio, fue de 11-12 años.

En cuanto al número y porcentaje de estudiantes, 6 de los 44 estudiantes de la muestra $(13,7 \%)$ presentarían déficit de atención, 4 hiperactividad más déficit de atención $(9,1 \%)$, seguidos de categorías más bajas.

En estos casos todos son varones y ninguna mujer, de lo cual se desprende que la relación niño-niña para el caso de déficit de atención es de 6:0 y que la relación niño-niña para déficit de atención más hiperactividad es de 4:0, siendo éstas proporciones poco mayores al 4:1 para poblaciones no clínicas que señalan los estudios internacionales como el de Blázquez, et al (2005). En el análisis de la tabla II.

Los casos se remitieron a una Profesional en Psicología particular, quien aplicó y valoró mediante entrevistas personalizadas, baterías y pruebas complementarias y tras este reconocimiento concluyó que 3 de ellos (los estudiantes números 3,4 y 6) presentan déficit de atención, no así los 3 restantes que sin embargo "si presentan altas puntuaciones en las escalas de valoración, no presentan déficit de atención ni significativo peligro en sus desarrollos cognitivos", según argumenta en su informe.

Por tanto, luego del reconocimiento de la profesional, 3 estudiantes presentan déficit de atención, que representan el 6,8\% del total de la muestra y que se ubica dentro de los rangos que señalan la mayoría de estudios para sujetos de poblaciones escolares y luego de diagnósticos más profundos; estudios como el de Hidalgo y Sánchez (2014) que señala del 5 al 7\%. 
La media de la variable "niveles de aprendizaje" como el consolidado de las medias entre rendimiento académico y dominio de conocimientos específicos es de 5,24/ 10 es decir 52,4\%, que al ubicarse debajo del punto de corte, se concluye que el niveles de aprendizaje del grupo es bajo.

El bajo nivel de aprendizaje de todo el grupo investigado se ubica entonces en $47,6 \%$, que es alto si consideramos que se encuentra dentro del rango 15 al $60 \%$ que autores como Blásquez, et al (2005) sostienen como dificultades de aprendizaje para alumnos con el trastorno de déficit de atención.

\section{CONCLUSIONES}

Las conclusiones a las que se llegaron en el estudio fueron que:

Los trastornos asociados a la atención que con más frecuencia se presentaron en el grupo investigado, según el test aplicado, y de manera general fueron: Déficit de atención, hiperactividad más déficit de atención, hiperactividad más déficit de atención más trastorno de conducta, hiperactividad y trastorno de conducta.

Los estudiantes que presentaron déficit de atención e hiperactividad más déficit de atención. En estos casos todos fueron varones.

Tres estudiantes presentaron el trastorno de déficit de atención y cuatro tienen bajo rendimiento escolar.

Los niveles de aprendizaje fueron estadísticamente significativos, siendo el rendimiento en matemática menor al de lenguaje.

Los niveles de aprendizaje, expresados en el estadístico, dominio de "conocimientos específicos", al contrario del rendimiento académico, muestran un resultado muy bajo. Así todos los estudiantes estuvieron por debajo de la media, siendo el dominio de conocimientos específicos en matemática es más bajo que en lenguaje.
Todos los estudiantes con déficit de atención, tienen bajo dominio de "conocimientos específicos".

\section{REFERENCIAS BIBLIOGRÁFICAS}

Chavarría., (2007). Educación en un mundo globalizado: retos y tendencias del proceso educativo. México: Editorial Trillas.

Kirby, E., Grimley, L., (1992).Trastorno por déficit de atención, estudio y tratamiento (primera edición). México: Editorial Limusa

Lahey, B.B.; Schaughency, E.A.; Frame, C.L.; Strauss, C.C. (1984). Are attention déficit disorder with and without hyperactivity similar o dissimilar disorders?. Journal of the American Academy of Child Psychiatry (núm. 23).

Blásquez-Almería, G., Joseph-Muné, D., Burón-Musó, E., Carrillo-Gonzáles, C., Joseph-Muné, M., Cuyás- Reguera, M., Freile-Sánchez, R. (2005), Resultados del cribado de la sintomatología del trastorno por déficit de atención con o sin hiperactividad en el ámbito escolar mediante la escala $E D A H$, Revista de Neurología (núm.41). Barcelona, España.

Triola, M. (2013). Estadística, actualización tecnológica (decimoprimera edición). México. Editorial Pearson.

Unidad Educativa Marista "Pío XII", (2013).Proyecto Educativo Institucional (Documento en construcción). Santo Domingo de los Tsáchilas.

\section{LINKOGRÁFICAS}

Martí, G., (2009). El déficit de atención. (En línea), disponible en http://www.psicologoescolar.com/MONOGRAFIAS/denia_gloria_marti_deficit_de_atencion.htm

Sánchez, E., (1998). Las dificultades de atención. ( En línea), disponible en http://www.galeon.com/criisthellopez/ libro2.pdf

Mañas, I., (2009). Mindfulness (Atención plena): La meditación en psicología clínica. (En línea), disponible en http://enelahora.com/2011/09/mindfulness-la-meditacion-en-psicologia-clinica/

Gamboa., A., (2009). Las adaptaciones curriculares. (En línea), disponible en http://es.slideshare.net/andrea_badilla/necesidades-especiales-y-las-tic

Ministerio de Educación del Ecuador (2015), Guía para Docentes de Educación General Básica. (En línea), disponible en http://educacion.gob.ec/ 